Itinéraires Itinéraires

Littérature, textes, cultures

2012-3 | 2013

Lire les villes marocaines

\title{
Yto Barrada : figures de résistance à la domestication de l'espace
}

\section{Évelyne Toussaint}

\section{(2) OpenEdition}

\section{Journals}

Édition électronique

URL : http://journals.openedition.org/itineraires/949

DOI : 10.4000/itineraires.949

ISSN : 2427-920X

Éditeur

Pléiade

\section{Édition imprimée}

Date de publication : 1 juillet 2013

Pagination : 57-67

ISBN : 978-2-343-01183-7

ISSN : $2100-1340$

\section{Référence électronique}

Évelyne Toussaint, «Yto Barrada : figures de résistance à la domestication de l'espace », Itinéraires [En ligne], 2012-3 | 2013, mis en ligne le 01 décembre 2012, consulté le 19 avril 2019. URL : http:// journals.openedition.org/itineraires/949; DOI : 10.4000/itineraires.949

\section{(C) $(\oplus \Theta$}

Itinéraires est mis à disposition selon les termes de la licence Creative Commons Attribution - Pas d'Utilisation Commerciale - Pas de Modification 4.0 International. 


\title{
Yto Barrada : figures de résistance à la domestication de l'espace
}

\begin{abstract}
The landscape is often the ground of politics, marked by power, hegemony and daily resistance to them. Yto Barrada bases her artistic approach on the observation of what she calls "resistance to the domestication of space." With a keen eye and a claimed tenderness, she describes not only the "property emergency" and "auto-folklorization" of the city of Tangier, but also its interstices of freedom.
\end{abstract}

Keywords : landscape, politics, resistance, space, Tangier Mots clés : paysage, politique, résistance, espace, Tanger

Yto Barrada est marocaine et française. Elle est née en 1971 à Paris où ses parents étaient étudiants. Elle a passé une partie de son enfance à Tanger où son père, Hamid Barrada, ancien opposant politique à Hassan II, leader de la gauche étudiante, est alors journaliste et sa mère, Mounira Bouzid, psychothérapeute. Plus tard, elle étudie l'histoire et les sciences politiques à la Sorbonne, puis la photographie à l'International Center of Photography de New York. Son intérêt pour le visuel s'est en effet imposé, explique-t-elle, quand elle a découvert que l'image, l'art, lui permettraient, mieux encore qu'une thèse de sciences politiques, de rendre compte de la guerre en Cisjordanie en photographiant les barrages militaires ${ }^{1}$. Ce travail fera l'objet, à l'Institut du monde arabe à Paris en 1998, d'une exposition dont Yto Barrada dit aujourd'hui qu'elle était sans grand intérêt esthétique mais qu'elle avait eu le mérite de l'avoir fait réfléchir et « sortir de ce type d'expositions essentialistes ${ }^{2} \gg$.

1. Yto Barrada et Charlotte Collins, « Morocco Unbound: an interview with Yto Barrada », Open Democracy, free thinking of the world, 16 mai 2006, http://www.opendemocracy.net/ arts-photography/barrada_3551.jsp (consulté le 5 avril 2013).

2. Courriel d'Yto Barrada à Évelyne Toussaint, le 10 février 2010. 
« Revenue à la maison » en 1999, elle vit à Tanger où, parlant trois langues, mariée à un Américain, elle affirme son cosmopolitisme et crée, en 2005, la Cinémathèque ${ }^{3}$. Elle est désormais reconnue en tant qu'artiste et participe à de nombreuses expositions dans le monde, ses œuvres étant notamment présentées lors de l'exposition «Africa Remix. L'art contemporain d'un continent » (2004-2007) mais aussi au Jeu de Paume à Paris en 2006 (Le Projet du Détroit), ou encore dans le cadre de la Biennale de Venise en 2007 et 2011, ainsi qu'au Centre Pompidou (« Elles@centrePompidou », 2009-2011) et à l'ICP de New York (Third Triennal of Photography and Video, 2010). Conviée par le commissaire général Okwui Enwezor à prendre part à la Triennale 2012 «Intense Proximité. Une anthologie du proche et du lointain » au Palais de Tokyo, elle y présente Hand-Me-Downs (2011), montage de films familiaux, anonymes, des années 1960, où se nouent l'intime et la mémoire collective. L'écriture accompagne un travail sur l'image par lequel elle s'intéresse aux mutations du Maroc, et plus particulièrement de Tanger, dans un contexte postcolonial, entre autonomie et nouvelles formes de dépendance ${ }^{4}$. Son regard n'est pas seulement critique, il est aussi sensible, en une approche phénoménologique des personnes et des lieux. Elle donne à voir, en artiste plasticienne et non en urbaniste ou en architecte, sa propre expérience poétique de la ville et de son environnement, retenant des images de lieux où se mêlent continuités et ruptures, mélancolie et espoirs, désirs et frustrations, ennui et petites occupations du quotidien, solidarités et exclusions.

\section{Art et bio-politique de la ville}

Une vie pleine de trous. Le Projet du Détroit est un ensemble d'œuvres vidéo, d'installations et de photographies relatives au détroit de Gibraltar. Dans ce work in progress entrepris en 1998, Y to Barrada donne à voir les habitants de ce territoire de l'entre-deux alors qu'ils s'adonnent à des activités illégales (contrebande de cigarettes pour Bornes ou de vêtements pour La Contrebandière), quand un travail quasi carcéral dans des usines occidentales les assujettit (Usine 1 - Conditionnement de crevettes dans la zone franche, 1998), ou encore lorsqu'ils sont, dans leur désœuvrement, comme absents à eux-mêmes (les Dormeurs, 2006, de la série Jardins Publics).

3. Le site de la galerie Polaris est largement documenté : http://www.galeriepolaris.com/ artistes.php?id=3; le site de l'artiste est disponible à l'adresse suivante : http://www.ytobarrada.com (consulté le 5 avril 2013).

4. Yto Barrada renvoie aux travaux de Sylvaine Bulle, sociologue et historienne, qui conduit des recherches sur la transcription urbaine des faits contemporains, notamment dans les métropoles du Sud : Dakar, Le Caire, Jérusalem, Téhéran, Rio de Janeiro (courriel d'Y to Barrada à Évelyne Toussaint, le 10 février 2010). 
C'est là, explique-t-elle, ce à quoi elle assiste, « ce qu'elle voit de sa fenêtre » : une frontière directement observable, des discussions sans fin sur la Méditerranée - «mère de toutes les mers »-, des gestes de partage et d'amitié, dans un lieu ouvert qui accueille des millions de touristes et où les antennes satellites laissent affluer, par des dizaines de chaînes, les images de l'Ouest, alors même que la grande majorité des gens ne peuvent légalement pas, depuis les accords de Schengen en 1991, et malgré leur désir, quitter le pays 5 .

Dès lors, le Détroit devient un mythe que des milliers de gens tentent de transformer en réalité pour gagner l'autre rive et la liberté. Cette obsession, insiste-t-elle, reste constamment présente dans le port de Tanger et c'est cela que montrent ses portraits : des gens qui attendent leur tour, souvent de dos, comme ils tournent le dos à leur pays, distraits de leur présent par le fantasme de leur impossible évasion, alors que le détroit de Gibraltar est désormais un espace pour clandestins et demandeurs d'asile ${ }^{6}$.

Y to Barrada - à qui sa double nationalité et son " pouvoir d'achat », comme elle le sait bien, autorisent une rare liberté - montre par ses images polysémiques, apparemment neutres, faussement banales, une ville de transition entre l'Afrique et l'Occident, une ville, dont la Beat Generation, avec Paul Bowles, avait confirmé la vocation littéraire et poétique, et qui se trouve aujourd'hui comme dépossédée de ses ancrages. Tanger est aujourd'hui devenue, écrit Yto Barrada, ce " grand cimetière marocain » dont le nom révèle la double face : « en arabe comme en français, détroit conjugue étroitesse (dayq) et détresse (mutadayeq) $)^{7} »$.

La première partie du titre qu'elle donne à cette œuvre, Une vie pleine de trous, est empruntée au livre de Driss Ben Hamed Charhadi, premier conteur marocain transcrit par Paul Bowles ${ }^{8}$, dont elle cite un passage : «On dit qu'il vaut mieux ne pas avoir de vie qu'une vie pleine de trous. Mais on dit aussi : mieux vaut un sac vide que pas de sac du tout. Je ne sais pas $^{9} . »$

La démarche plasticienne d'Y to Barrada, dont Le Projet du Détroit est particulièrement emblématique, aborde ainsi poétiquement des questions d'ordre géopolitique. Elle interroge aussi, selon une approche quasi anthropologique, des problématiques collectives en lien avec des politiques de

5. «Morocco Unbound: an interview with Yto Barrada», op. cit.

6. Ibid.

7. «Y Yto Barrada », Jeu de paume, http://www.jeudepaume.org/?page=document\&idArt $=151 \&$ lieu $=2 \& \mathrm{idDoc}=244 \&$ PHPSESSID=077bdbe761fe878f96bd13a797 (consulté le 5 avril 2013).

8. Driss Ben Hamed Charhadi, Une vie pleine de trous, recueilli et transcrit par Paul Bowles, trad. de l'anglais par Céline Zins, Paris, Gallimard, 1965.

9. Entretien avec Nadia Tazi, dans A Life Full of Holes, The Strait Project, Londres, Autograph ABP, 2005; http://www.ytobarrada.com/press/JeuDePaume.pdf (consulté le 5 avril 2013). 
gestion de l'urbanisme et des stratégies immobilières imposant la mutation accélérée des paysages.

La maquette animée (Gran Royal Turismo, 2003) qu'elle présente dans le cadre d' «Africa Remix » puis lors de l'exposition « Disorientation II » à Abu Dhabi, montre un Tanger de façade, où les palmiers sortent de terre au passage d'un convoi officiel de trois Mercedes à l'échelle 1/45 :

Immobilisés par respect, par peur ou par besoin de merveilleux, [les habitants] sont volontairement ignorés dans Gran Royal Turismo. Ce Maroc éternel et immobile renvoie à une idée liée à la représentation du «plus beau pays au monde » faite par l'industrie du tourisme et valorisée auprès des sujets du Royaume. De cette auto-folklorisation chère aux Marocains découle une incapacité à penser le pays autrement ${ }^{10}$.

Cette installation satirique fonctionne - ce dont rendent parfaitement compte les propos de l'artiste -, sur le mode du Witz, du mot d'esprit, procédant, comme l'expliquait Freud, par condensation (une seule image concentrant plusieurs idées et représentations) et déplacement (une représentation à la place d'une autre, ici les palmiers, en place des habitants, aux ordres du passage officiel). Les photographies, les vidéos et les installations d'Y to Barrada jouent ainsi souvent sur le registre de la condensation, structure, selon Lacan, d'une « surimposition des signifiants où prend son champ la métaphore ${ }^{11} »$, les œuvres entrant de plus en résonance les unes avec les autres. Gran Royal Turismo se trouve par exemple en lien avec les photographies de la série Iris Tingitana (2007).

Autour de Tanger, le développement économique et la spéculation immobilière transforment l'urbanisme, les constructions effrénées investissant les espaces de pâturage, les marchés, les forêts, les friches, les terrains vagues ou les plages, tandis que les bâtiments historiques sont impétueusement recyclés ${ }^{12}$. La flore locale - iris et autres fleurs sauvages $-\mathrm{s}$ 'en trouve menacée alors que les géraniums roses fluo et les palmiers importés pour les touristes envahissent le paysage, qui devient la Costa del Sol marocaine, à haute densité immobilière pour tourisme de masse ${ }^{13}$. Or, l'iris, fragilisé mais insistant, survit - certes, peut-être provisoirement - à l'inflation urbaine et à l'anthropisation des lieux. La série Iris Tingitana témoigne donc d' « une stratégie de résistance active à la domestication d'une ville ${ }^{14} \gg$.

Yto Barrada met ici en perspective le monumental et le détail, la force et la fragilité : « En cherchant la bonne distance, je voulais voir d'un autre

10. Y to Barrada, Le Détroit ou une vie pleine de trous, documentation de l'artiste transmise par la galerie Polaris, dirigée par Bernard Utudjian.

11. Jacques Lacan, Écrits, Paris, Seuil, 1966, p. 511.

12. Ce que traduit, par exemple, l'œuvre : Vacant lot 2, série Iris Tingitana, rue de Fez, Tanger, 2007.

13. http://www.ytobarrada.com/press/Barrada_Biennale.pdf(consulté le 5 avril 2013).

14. http://www.ytobarrada.com/press/ComingUP-IrisTingitana.pdf(consulté le 5 avril 2013). 
point de vue les grands chantiers qui dévorent la ville. Je suis partie de comparaisons de cartes et de photographies aériennes (infiniment grand) et de catalogues de botanique et de faune d'Afrique du Nord (infiniment petit). » Son constat est sévère quant aux causes et aux conséquences de ce qu'elle appelle « la botanique du pouvoir » :

La conquête de Tanger, lancée en grande pompe par les autorités il y a cinq ans, a conduit à la construction d'un décor de carton conçu par des gens qui connaissent bien la technique du béton armé mais qui ignorent tout de l'histoire de l'art, des peuples et du paysage ${ }^{15}$.

L'idée de «botanique du pouvoir » renvoie à la pensée de Michel Foucault, et l'on se souviendra ici avec lui qu'il existe une bio-politique, et que « le contrôle de la société sur les individus ne s'effectue pas seulement par la conscience ou par l'idéologie, mais aussi dans le corps et avec le $\operatorname{corps}^{16} \gg$.

Lors de la présentation de ce travail à la galerie Polaris, à Paris, Yto Barrada a offert aux visiteurs de petites «bombes à graines » à disséminer : « Dans ce rapport de force, la tactique de la guérilla, c'est-à-dire de l'effet de surprise, peut être un avantage ${ }^{17}$. » Cette action peut évoquer celle de Liz Christy, créatrice du premier jardin communautaire de New York, inventrice, au début des années 1970, du terme " green guerilla », qui utilisait des bombes de graines et de terreau à jeter par-dessus les palissades des terrains vagues. Le geste d'Yto Barrada s'inscrit dans la lignée de plusieurs actions conduites par des artistes du Land-art (herman de vries ${ }^{18}$, par exemple) ainsi que par des militants écologistes un peu partout dans le monde mais son « inspiration/filiation, précise-t-elle, est surtout celle des Diggers anglais du XVII ${ }^{\mathrm{e}}$ siècle, peut-être les premiers squatteurs, et de ceux de San Francisco dans les années 1960, ces derniers appartenant à la contre-culture anarchiste ${ }^{19} \gg$.

Dans le même esprit de préservation de la nature contre les constructions outrancières, elle a réalisé en 2009 un film en $16 \mathrm{~mm}$, Beau Geste, notamment programmé en 2010 par la galerie Sfeir Semler à Beyrouth ${ }^{20}$.

15. Pour les deux citations de ce paragraphe: Yto Barrada, citée par Claire Moulène, «Flower power. La folie immobilière a saisi Tanger. Documentée par Yto Barrada: une ode à la résistance métaphorique des fleurs dans la ville défigurée », Les Inrockuptibles, 17 octobre 2007.

16. Michel Foucault, «La naissance de la médecine sociale», dans Dits et Écrits, II, 1976-1988, Paris, Gallimard, 2001, p. 210.

17. Yto Barrada, citée par Claire Moulène, op. cit.

18. Je respecte ici la volonté de l'artiste de voir son nom toujours écrit en lettres minuscules.

19. Yto Barrada, citée par Claire Moulène, op. cit.

20. http://www.sfeir-semler.de/last/AdnanBarrada/AdnanBarrada_2010.htm (consulté le 5 avril 2013). 
Ici, il s'agit de sauver les palmiers, abattus au rythme frénétique de la distribution des permis de construire.

Ces beaux et petits gestes, que propose Yto Barrada pour réinventer le quotidien, ressemblent bien à une tactique, dont Michel de Certeau écrit qu'il lui faut utiliser « les failles que les conjonctures particulières ouvrent dans la surveillance du pouvoir propriétaire. Elle y braconne. Elle y crée des surprises. Il lui est possible d'être là où on ne l'attend pas. Elle est ruse. En somme, c'est un art du faible ${ }^{21} »$. Et cet « art du faible » n'exclut pas de s'emparer des problèmes les plus complexes, qu'ils soient politiques ou philosophiques.

\section{De l'exotisme à l'anthropologie réciproque : une traduction plastique}

Ces trois axes de travail procèdent, sous des angles différents (du frontalement critique Gran Royal Turismo à la démarche politico-philosophique d'Iris Tingitana, en passant par la banque d'images à la fois documentaire et poétique d'Une vie pleine de trous. Le Projet du Détroit), d'un questionnement sur les mutations apparentes de la ville contemporaine, ses infrastructures et ses métamorphoses architecturales et urbanistiques, alors que dans le même temps, le désir de départ est l'un des symptômes de la perte de sens vécue par les habitants.

Mettant en évidence un espace où se confrontent enracinement et tentation de déterritorialisation, Yto Barrada montre Tanger en tant qu'espace clivé, où se nouent d'irréductibles contradictions : la proximité des côtes espagnoles (une quinzaine de kilomètres) et le fait que cette faible distance soit infranchissable; l'ouverture du pays au tourisme de masse et la fermeture au monde vécue par ses habitants; les emblèmes de la tradition qui construisent une identité-vitrine et l'inflation d'images du monde qui muséifient cette tradition en marge du mainstream des technologies, des mutations économiques, sociales, culturelles et religieuses, de New York à Dubaï; une ville mythique, imaginaire, et la banalité du quotidien... De ces contradictions naît une ambivalence permanente entre ouverture et repli, ancrage et désir de départ, dans la complexité des motivations collectives et individuelles.

Pour Gilles Deleuze et Félix Guattari, la déterritorialisation de la pensée est un moment de déconditionnement, de désassujettissement, la chance de pouvoir effectuer des choix et procéder à des ruptures sélectives. Au fil de leurs écrits, parce qu'ils ont toujours refusé qu'ils fassent office de système holistique de pensée, l'idée de déterritorialisation prend des acceptions différentes mais, dans Qu'est-ce que la philosophie?, ils conçoivent la déterritorialisation « absolue » comme s'inscrivant dans « le pur plan

21. Michel de Certeau, L'Invention du quotidien, t. I, Arts de faire, Paris, Gallimard, 1990, p. 61 . 
d'immanence d'une pensée ${ }^{22} \gg$. La déterritorialisation psychologique ne se réduit donc pas à une déterritorialisation géographique, d'autant que, comme ils l'écrivaient déjà dans L'Anti-CEdipe, « il est impossible de distinguer la déterritorialisation et re-territorialisation, qui sont prises l'une dans l'autre ou sont comme l'envers et l'endroit d'un même processus ${ }^{23} »$. La démarche esthétique (c'est-à-dire artistique et éthique) d'Y to Barrada s'inscrit, au final, dans cette logique : à tourner le dos à leur passé, les hommes dont les photographies d'Yto Barrada fixent l'image, sont en danger d'être ravis - au sens où le fut Lol V. Stein dans le roman de Marguerite Duras ${ }^{24}$ à leur présent, emportés dans la représentation d'un ailleurs impossible, tandis que s'anéantissent leur vie comme leur territoire.

Revenant elle aussi, comme de nombreux artistes contemporains, sur la problématique des représentations et des préjugés dont elles témoignent, Yto Barrada aborde tout aussi efficacement la question de l'exotisme, en photographiant simplement un papier peint figurant un paysage alpin, décorant le mur d'un $\operatorname{bar}^{25}$. Quand Tanger se réduit, pour les Occidentaux, au cliché de la "Ville blanche » (image en laquelle se résumeraient les visions orientalistes traditionnelles et leur actualisation confirmée par n'importe quel site touristique sur Internet aujourd'hui, à grand renfort de femmes lascives, de thé à la menthe et de géraniums roses), une photographie des Alpes enneigées rendrait assez bien compte de ce que peut être un paysage exotique pour des Marocains.

Y to Barrada apporte ainsi une touche très personnelle à l'idée controversée d'exotisme, selon une approche transculturelle et, en quelque sorte, post-exotique. On pourrait ici évoquer l'idée d'une anthropologie réciproque, chère, notamment, à Alain Le Pichon, fondateur, avec Umberto Eco, de l'institut Transcultura «pour une reconnaissance réciproque des civilisations ${ }^{26} »$. Dès lors, on comprend mieux la volonté de l'artiste d'en finir, dès ses débuts, avec des « expositions essentialistes ».

Dans un contexte postcolonial où la réciprocité pourrait enfin advenir en place d'un certain exotisme unilatéral, souvent condescendant, de nouvelles modalités sont à inventer qui supposent la démocratisation de la culture, garante d'ouverture au monde, de distance critique et de créativité. C'est dans cet esprit que l'un des premiers projets d'Y to Barrada, Fais un

22. Gilles Deleuze et Félix Guattari, Qu'est-ce que la philosophie?, Paris, Minuit, 1991, p. 85 .

23. Gilles Deleuze et Félix Guattari, Capitalisme et schizophrénie. L'Anti-CEdipe [1972], Paris, Minuit, 1991, p. 307.

24. Marguerite Duras, Le Ravissement de Lol V. Stein [1964], Paris, Gallimard, 1976.

25. Une vie pleine de trous. Le projet du détroit, papier peint, photographie en couleur, $60 \times 60 \mathrm{~cm}, 2001$.

26. Alain Le Pichon a fondé, avec Daiyun Yue, les principes d'une anthropologie réciproque : Alain Le Pichon et Yue Daiyun, La Licorne et le Dragon: les malentendus dans la recherche de l'universel, Paris, Charles Léopold Mayer, 2003. 
fils et jette-le à la mer, entendait instaurer des échanges entre des enfants de l'association Jeunes errants à Marseille et des enfants des rues de Tanger, en leur proposant simultanément des ateliers de photographies donnant lieu à des échanges de vues de leurs villes respectives ${ }^{27}$. La création de la Cinémathèque, à Tanger, s'inscrivait dans la même logique d'ouverture et de découverte d'images venant du monde entier, libérant la parole collective, permettant d'envisager le présent sous l'angle du possible et autorisant le partage de singularités.

Cette posture éthique se traduit plastiquement dans le travail d'Y to Barrada qui reconnait une source d'inspiration dans le regard scrutateur et documentaire de Walker Evans (1903-1975) ${ }^{28}$. Partageant son attention à la personne et son intérêt pour le contexte collectif, son goût pour le quotidien et la dimension universelle dont il est porteur et qu'un regard attentif révèle, elle cherche à son tour à donner à voir, comme elle l'explique, « la tension entre l'allégorie et l'instantané ${ }^{29}$ ».

Comme jamais, dans les arts plastiques, la forme n'est dissociable du sens, Yto Barrada évite la construction unitaire et toute systématisation globalisante, préférant l'idée de photomontage à la linéarité du récit : «On trouve des motifs récurrents dans mes photographies, platitude des tons, perte de l'épaisseur des choses (volumes écrasés, murs omniprésents, etc.), mais mon travail s'est construit comme une juxtaposition d'images différentes ${ }^{30}$. » Ce parti pris permet à l'artiste d'élaborer un dispositif d'énonciation ouvert, fragmentaire, non autoritaire et à même de proposer une nouvelle lecture de la ville, postcoloniale et post-exotique, à l'écart des stéréotypes.

Dans un article ayant pour titre " Postcolonialisme et comparatisme", Jean-Marc Moura retient divers modes d'interprétation « enracinés dans les études postcoloniales » : une perspective historique, sous l'angle d'une histoire transnationale et de l'intérêt pour les « minorités »; une perspective interculturelle, s'attachant au caractère hybride de nos sociétés; une poétique, les œuvres s'inscrivant «dans une situation d'énonciation où coexistent des univers symboliques divers dont l'un a d'abord été imposé et a reçu le statut de modèle ou contre lequel on s'affirme »; la prise en compte du « sentiment de la langue ${ }^{31} »$. Toutes ces modalités se trouvent

27. Cette action a donné lieu à la publication d'un livre : Yto Barrada, Anaïs Masson et Maxence Rifflet, Fais un fils et jette-le à la mer : Marseille-Tanger : une expérience d'ateliers photographiques avec des adolescents des associations Jeunes errants (Marseille) et Darna (Tanger), récit et montage, Paris, Sujet-Objet, 2003.

28. Rasha Salti, «Sleepers, Magicians, Smugglers: Yto Barrada and the Other Archive of the Strait », Afterall, $n^{\circ} 16,2007$. Disponible en version imprimée dans le dossier de l'artiste consultable à la galerie Polaris, Paris.

29. «Y to Barada », Jeu de paume, op. cit.

30. Ibid.

31. Jean-Marc Moura, «Postcolonialisme et comparatisme », Vox Poetica, http://www. vox-poetica.org/sflgc/biblio/moura.html, publié le 20 mai 2006 (consulté le 5 avril 2013). 
réunies dans la démarche d'Y to Barrada, autant que la nécessité impérieuse, sur laquelle insiste Jean-Marc Moura, d'un point de vue transnational.

Ainsi, l'œuvre d'Yto Barrada donne en effet à voir et à penser, poétiquement et politiquement, la ville marocaine aujourd'hui. Entre liberté et emprise.

Évelyne Toussaint 


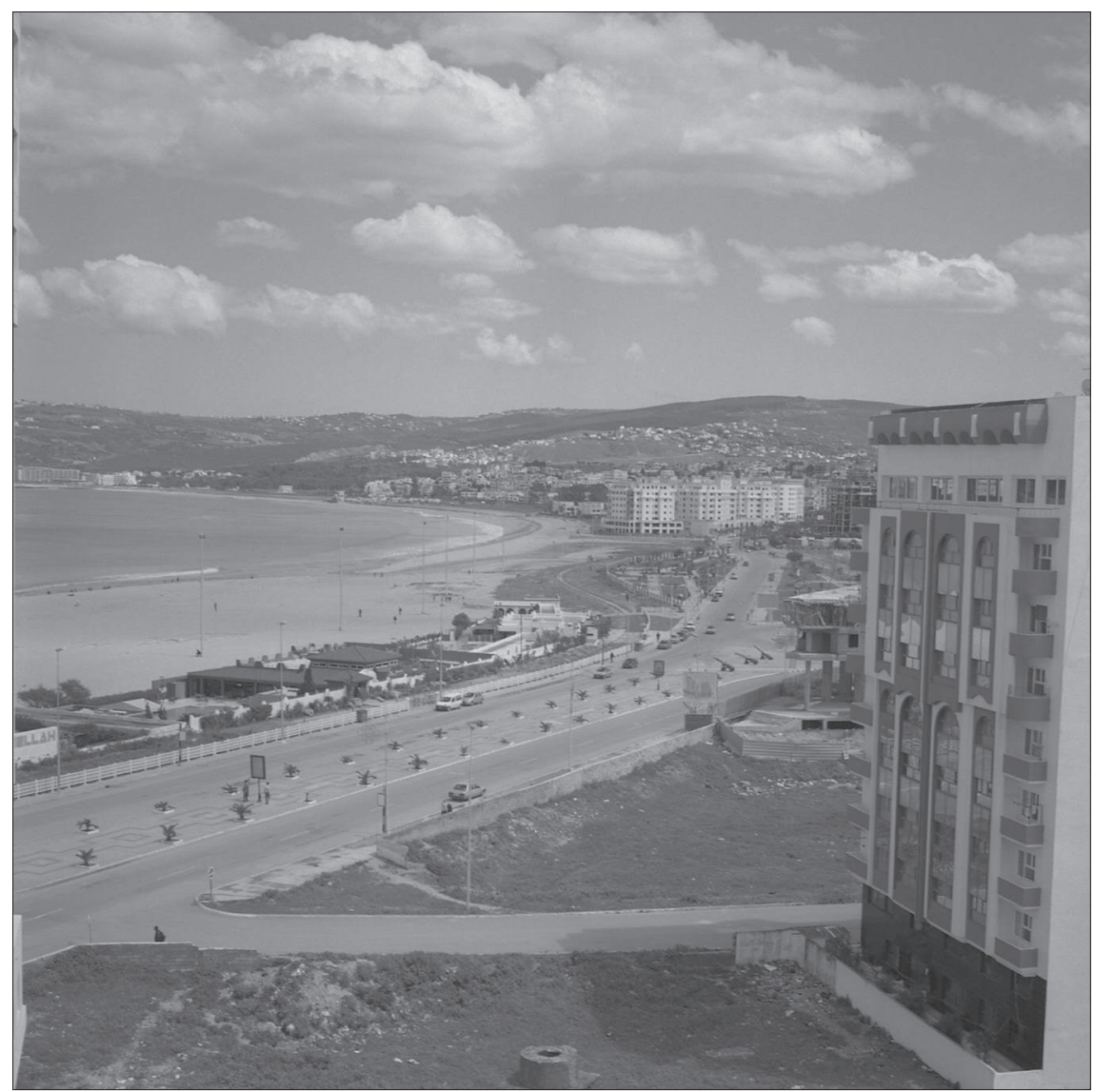

Yto Barrada, Vacant Lot 2, Tanger, de la série Iris Tingitana, 2009, C-Print, $100 \times 100 \mathrm{~cm}$ 


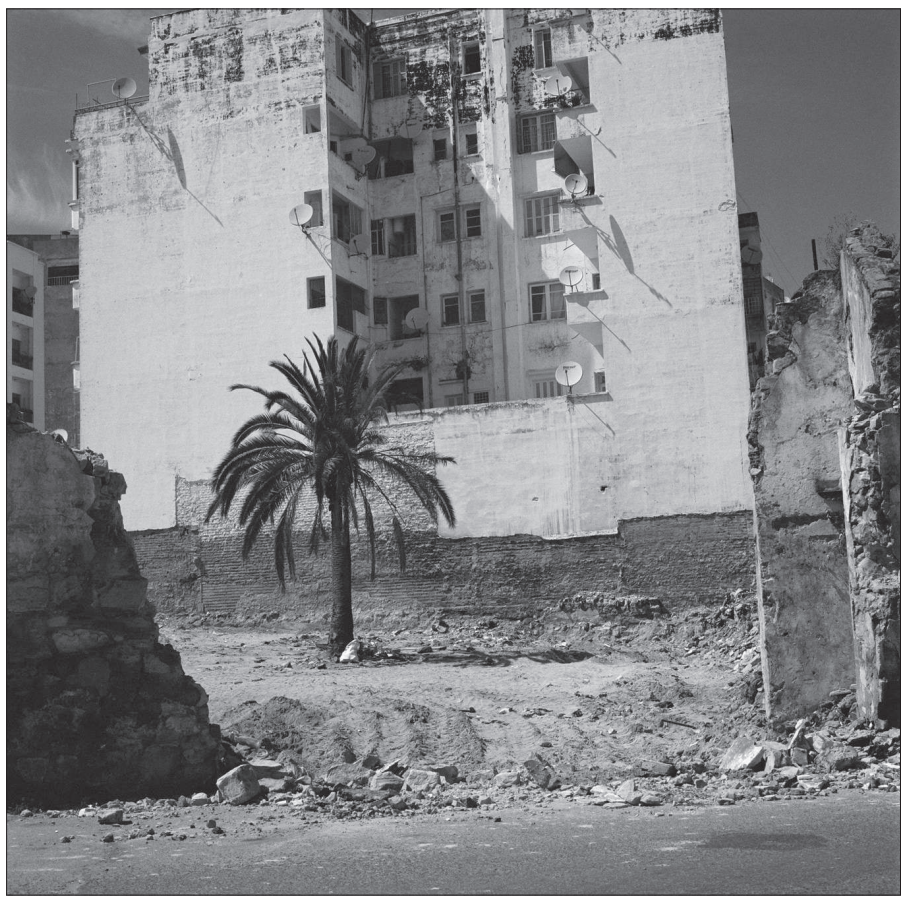

Yto Barrada, Papier peint, Tanger, de la série Le Projet du Détroit, 2001, C-Print, $60 \times 60 \mathrm{~cm}$

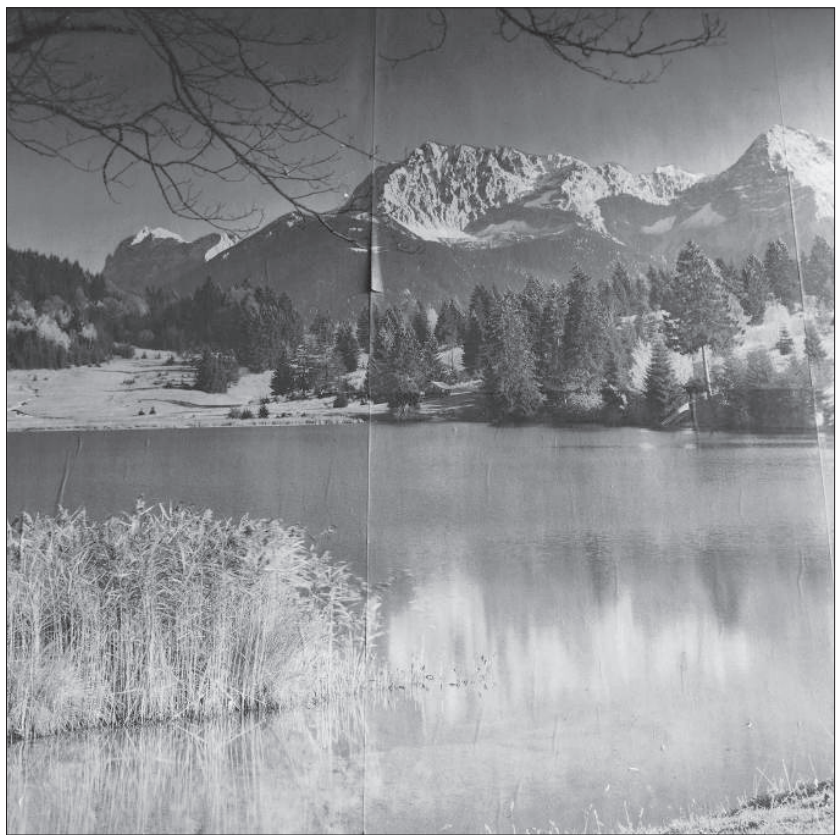

Yto Barrada, Baie de Tanger, de la série Le Projet du Détroit, 2002, C-Print, $60 \times 60 \mathrm{~cm}$ 